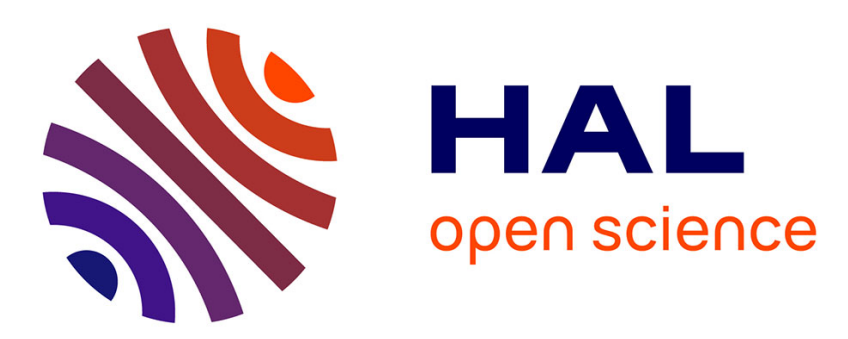

\title{
Personnifications, réifications et métaphores créatives dans le système rhétorique de Charles d'Orléans.
}

Claudio Galderisi

\section{To cite this version:}

Claudio Galderisi. Personnifications, réifications et métaphores créatives dans le système rhétorique de Charles d'Orléans.. Romania (Paris), 1996, CXIV (455), pp.385-412. 10.3406/roma.1996.2213 . halshs-00943440

\author{
HAL Id: halshs-00943440 \\ https://shs.hal.science/halshs-00943440
}

Submitted on 3 Mar 2017

HAL is a multi-disciplinary open access archive for the deposit and dissemination of scientific research documents, whether they are published or not. The documents may come from teaching and research institutions in France or abroad, or from public or private research centers.
L'archive ouverte pluridisciplinaire HAL, est destinée au dépôt et à la diffusion de documents scientifiques de niveau recherche, publiés ou non, émanant des établissements d'enseignement et de recherche français ou étrangers, des laboratoires publics ou privés. 
Personnifications, réifications et métaphores créatives dans le système rhétorique de Charles d'Orléans

Claudio Galderisi

\section{Citer ce document / Cite this document :}

Galderisi Claudio. Personnifications, réifications et métaphores créatives dans le système rhétorique de Charles d'Orléans. In: Romania, tome 114 n455-456, 1996. pp. 385-412;

doi : 10.3406/roma.1996.2213

http://www.persee.fr/doc/roma_0035-8029_1996_num_114_455_2213

Document généré le 16/11/2016 


\section{PERSONNIFICATIONS, REIFICATIONS ET MÉTAPHORES CRÉATIVES DANS LE SYSTÈME RHÉTORIQUE DE CHARLES D'ORLÉANS}

Dcpuis un sic̀cle, plus de quarante travaux et deux mille pages ont été consacrés au rôle de l'allégorie, de la personnification et de la métaphore - parfois sans aucune distinction formelle - dans les poèmes de Charles d'Orléans '. Si la critique semble, donc, avoir apprécié, et peut-être même surestimé, le caractère subjectif de l'imagerie aurélienne, finissant par en faire la clé d'interprétation, parfois quelque peu impressionniste, de son code poétique, il a manqué, en revanche - à l'exception de quelques travaux isolés ${ }^{2}$-,

1. Cf. C. Galderisi, Charles d'Orléans : "Plus dire que penser ", Bari, 1994, p. $52-61$.

2. Cf. P. Zumthor, Charles d'Orléans et le langage de l'allégorie, dans Mélanges Rita Lejeune, Gembloux, 1969, t. II, p. 1481-1502; Id., Essai de Poétique Médiévale, Paris, 1972, p. 278-85 ; D. Poirion, La Nef d'Esperance. Symbole et allégorie chez Charles d'Orléans, dans Mélanges Jean Frappier, Genève, 1970, t. II, p. 913-928 ; B. Turksma-Heijmann, Contribution à une théorie de l'allégorie, dans Rapports : Het Franse Boek, mars 1976, p. 37-47; A. Strubel, En la Forêt de Longue Attente. Réflexions sur le style allégorique 
non seulement une réflexion sur le mode de fonctionnement de ce système rhétorique, mais parfois jusqu'à la conscience critique de la nécessité d'une définition méthodologique des modes de production. Le système rhétorique courtois a été souvent pris comme modèle de référence - in absentia, on serait tenté de dire - , sans qu'aucun calibrage ou rajustement conceptuel spécifique ait été opéré pour donner une cohérence éditoriale aux termes à majuscule, sans qu'aucune définition préalable du procédé esthétique aurélien ait supporté les efforts herméneutiques.

Il faut ajouter, dès maintenant, qu'une telle attitude tenait dans quelques cas au refus conscient de considérer Ballades, Chansons et Rondeaux comme des véritables corpus poétiques constitués, avec une dimension esthétique propre, sinon un véritable plan romanesque et une unité narrative ${ }^{3}$. Une approche - mais dans quelques cas on serait plutôt tenté de parler d'une habitude - , où se fondaient idées reçues alimentées par le vieux jugement de $P$. Champion sur la facilité de la versification aurélienne et une esthétique "monadique " double moderne de la technique du commentaire de texte. Certes, Charles d'Orléans a été un poète qui a souvent charmé et enchanté ses exégètes, au point de les faire, parfois, céder à la tentation de le suivre sur son propre terrain : celui impraticable de la création poétique ${ }^{4}$. Mais vouloir théoriser, comme d'aucuns l'ont fait, l'absence ou le caractère insaisissable de toute structure supratextuelle dans son œuvre, et renoncer, ainsi, à tous les aperçus que la lexicologie ou la sémiotique peuvent ouvrir, et de fait ont ouvert, sur un texte comme celui du prince, s'avérerait être un «hara-kiri»

de Charles d'Orléans, dans Style et Valeurs. Pour une histoire de l'art littéraire au Moyen Age, éd. Daniel Poirion, Paris, 1990, p. 167-186.

3. Daniel Poirion a lucidement démêlé d'abord et patiemment renoués, ensuite, les fils de l'inspiration thématique dans les premiers poèmes du Duc, parvenant à reconstituer la structure narrative sous-jacente à l'élaboration poétique (D. Poirion, Création poétique et composition romanesque dans les premiers poèmes de Charles d'Orléans, dans Revue des Sciences Humaines, 90 [1958], p. 185-211).

4. Une perspective critique, celle-ci, consciemment annoncée par Alice Planche : "Charles d'Orléans est le type de l'artiste qu'on ne peut aimer sans tenter de participer à la génèse de sa création ", qui mène, toutefois, à une impasse critique (A. Planche, Charles d'Orléans ou la recherche d'un langage. Paris, 1975, p. 731). 
critique, que ne justifieraient ni les risques d'une systématisation aveugle ou de manière, ni les tentations modernes d'un déconstructionnisme fin en soi.

Il n'y a pas lieu. ici, d'élaborer un outil de décodification multidisciplinaire, et pour cause, en revanche, il nous paraît urgent de tenter de définir la forme et le(s) mode(s) de ce systc̀me rhétorique: essayer de mesurer son rôle dans la génèse poétique aurélienne. Cependant, un tel programme serait encore bien trop vaste et prétentieux, s'il n'y avait pas eu les efforts d'analyse et de classement organique de la matière accomplis par Daniel Poirion, Armand Strubel et Paul Zumthor, qui ont tous contribué à mettre au jour le fonctionnement linguistique et sémantique du langage allégorique aurélien, et à en définir la typologie. C'est grâce à ces travaux ${ }^{5}$ - mais aussi grâce aux recherches conduites par Pierre Champion et Gilbert Ouy sur la librairie de Charles d'Orléans ${ }^{6}$... que nous pouvons évaluer aujourd'hui, par exemple, l'intérêt que le Duc portait aux artes predicandi ${ }^{7}$ et à leurs trois niveaux de signification - littera, sensus et sententia -, et par conséquent mesurer l'influence de la poésie allégorique profane et surtout de l'exégèse biblique sur son système rhétorique. Toutefois, le mode allégorique aurélien, qui caractérise d'une manière ou d'une autre plus de $80 \%$ de ses poèmes ${ }^{8}$, ne peut être entièrement réduit à un

5. Sur l'évolution de la forme allégorique dans la poésie française médiévale, voir H. R. Jauss, Genèse de la poésie allégorique française au Moyen Age (1180-1240), Heidelberg, 1962.

6. Cf. P. Champion, La librairie de Charles d'Orléans, Paris, 1910, et G. Ouy, Recherches sur la librairie de Charles d'Orléans et de Jean d'Angoulême pendant leur captivité en Angleterre et étude de deux manuscrits autographes de Charles d'Orléans, dans Comptes rendus de l'Académie des Inscriptions et Belles-Lettres, avril-juin 1955, p. 273-288.

7. Charles d'Orléans avait, par exemple, dans sa bibliothèque, les œuvres de celui que l'on appela le nouvel Augustin : Hugues de Saint-Victor, et en particulier ce Didascalicon où le chanoine distingue, dans un véritable traité d'herméneutique, les trois sens de l'écriture : le sens littéral ou historique, qui est celui du récit et auquel il convient de donner sa juste importance, le sens allégorique fondement du sens théologique, et le sens tropologique qui permet de lire dans l'Écriture les enseignements spirituels et moraux destinés à orienter le comportement humain (cf. ms. B.N., lat. 2532).

8. D'après Paul Zumthor, le nombre total des "actions allégoriques ", 
épigone tardif, ou à une "aurore boréale " 9 de la tradition rhétorique médiévale, et, de ce fait, décodé automatiquement à travers ses règles et ses critères. Son fonctionnement est plus subtil et plus complexe, sa logique plus personnelle, son axe conceptuel plus introspectif, comme le prouve, d'ailleurs, le tableau des pratiques allégoriques auréliennes retracé par Armand Strubel.

Ce qui fait, sans doute, la particularité de ce système, ce n'est ni

dans l'œuvre de Charles d'Orléans, s'élèverait à 1137, alors que le nombre de personnages allégoriques ne dépasserait pas les 380 (cf. P. Zumthor, op. cit., p. 486-491). En l'absence d'une théorie de la grammaire poétique, nombre de ces cas apparaissent comme douteux, ce qui explique, aussi, les discordances, parfois importantes, entre les différents recensements allégoriques réalisés depuis plus d'un siècle par la critique aurélienne (cf. F. Kuhl, Die allégorie bei Charles d'Orléans [thèse], Marburg, 1886, qui relève 137 "allegorische Gestalten "; J.C. Mühlethäler, Charles d'Orléans. Ballades et Rondeaux, Paris, 1992, p. 776-780, qui en suivant la distinction proposée par Armand Strubel entre personnification et réification réduit le nombre de personnages à 88 ; C. Galderisi, Le lexique de Charles d'Orléans dans les Rondecaux, Genève, 1992, avec un relevé de 144 figures recensées dans les Ballades et dans les Rondeaux, fondé sur le choix de dénombrer séparément les substantifs et les adjectifs composant les syntagmes allégoriques). En effet, l'absence de déterminants actualisateurs devant les substantifs abstraits ou désignant une notion générale, avec une fonction de sujet, mais aussi d'objet, encore largement attestéc en $\mathrm{MF}$, ne peut que rendre incertaine toute tentative de classement du procédé allégorique qui se fonderait sur une discriminante purement grammaticale. Dans un bref sondage réalisé par Christiane Marchello-Nizia, il résulte que, sur cent substantifs choisis au hasard dans les poèmes auréliens, quarante-quatre ne présentent pas de déterminant, sans que cette absence autorise pour autant à parler de personnifications (cf. C. Marchello-Nizia, Histoire de la langue française au $X I V^{r}$ et $X V^{r}$ siecles, Paris, 1979, p. 112).

9. Dans une communication faite au colloque de Provins, sur la notion d'apogée et de déclin au Moyen Âge, j'avais proposé d'interpréter le rondeau aurélien comme une aurore boréale de la littérature courtoise, qui aurait été provoquée par un "phénomène de sublimation par contamination" au contact d'un monde et d'un lexique nouveau. Cette formule suggestive, qui avait peut-être le mérite de poser le problème des résurgences formelles et thématiques, me paraît aujourd'hui inadéquate à expliquer le caractère authentique du message poétique aurélien (cf. C. Galderisi, Les Rondeaux de Charles d'Orléans : une aurore boréale de la poésie courtoise, dans Apogée et Déclin, éd. Claude Thomasset et Michel Zink, Paris, 1993, p. 249-256). 
la sélection des abstracta agentia, qui à l'exception de Nonchaloir, Ennui, et en partie Mélancolie, ne présentent pas un caractère particulièrement original, ni l'utilisation de ces personnifications "homéopathiques" en doses massives, mais le rôle rythmique et syntaxique "d'agitateurs de réflexions" que le Duc confie à ces véritables holorimes de la conscience, et, surtout, une délittéralisation du contexte poétique, opérée à travers un renouveau, sans précédent à l'intérieur du microcosme courtois, du lexique poétique ${ }^{10}$, qui enlèvent aux abstracta agentia toute connotation impersonnelle, en les enracinant dans la dimension psychologique du poète, à travers le contact sémantique, la contamination idéologique avec les realia. C'est cette nature à la fois physique et conceptuelle de son système d'images, qui permet au poète de s'identifier à tour de rôle avec ces figures poétiques, d'accéder au virtuel poétique, en amoncelant d'abord, et en le diluant ensuite son fardeau humain dans ces lexèmes à la vertu thaumaturgique. Mais quels sont les paramètres qui permettent au poète d'opérer ce transfert ? Quels sont les principes logiques qui gouvernent ou rendent possible une telle osmose?

La notion d'«action allégorique» proposée par P. Zumthor semble bien offrir un instrument d'investigation de ce lien entre monde physique - où significations vécues et concepts connus se rejoignent -- et mondes possibles, c'est-à-dire non pas l'ailleurs du rêve, mais celui de la pensée. Le procédé narratif par séquences d'images semble convenir parfaitement, en effet, à la narration hoquetée de la ballade et surtout du rondeau. Mais peut-on essayer de définir une poétique de l'allégorie indépendamment d'une définition, sinon d'une codification, de la grammaire poétique ? $\mathrm{Ne}$ faudrait-il pas voir dans l'allégorie un des modes (sinon le mode) de cette grammaire poétique, et comme tel l'étudier? Il nous convient alors, ici, avant même d'avancer une définition de l'action allégorique et de ses différents modes de production, d'aborder la vexata questio des interférences entre langue et langage poétique (aurélien), donc entre syntaxe et versification.

En effet, l'enquête cognitive, la réflexion introspective semblent se

10. D. Poirion, Le lexique de Charles d'Orléans dans les Ballades, Genève, 1967, p. 15-27, et C. Galderisi, Le lexique de Charles d'Orléans dans les Rondeaux, op. cit., p. 43-58. 
concilier difficilement avec les règles sévères et tyranniques de la versification de la ballade et surtout du rondeau. L'espace clos du vers et de la strophe semblerait empêcher toute articulation de la pensée, donc toute possibilité élocutoire. Mais Charles d'Orléans est un poète qui a connu la prison, et sait donc bien, comme le suggère le poète anglais Richard Lovelace, que "stone wall do not a prison make/nor iron bars a cage ». Le Duc s'imagine, alors, heureux dans le cadre inconfortable du poème cyclique. Il cherche et parvient, ainsi, à vaincre la force centripète de la structure syntaxique de la langue, par la sonorité et la charge émotionnelle de mots combinés entre eux selon les normes inclassables du style, de son style. Le sentiment poétique court-circuite la concaténation normative à travers une condensation, une compression, de la pensée. La nouvelle organisation de la phrase poétique s'opère à travers une variation contextuelle du sème nucléaire du mot, par conséquent une redéfinition de sa nature quantitative ${ }^{11}$. Le poète s'attaque ainsi, par cette rupture des codes d'énonciation, à l'«inertie de la chose écrite ", lucidement évoquée par Valéry ${ }^{12}$.

Tout comme la voix peut dans certaines conditions ${ }^{13}$ être indépendante du langage, les mots du poète peuvent cacher pour mieux le révéier un pouvoir autonome, distinct de leur signification courante, qui échappe à toute analyse paradigmatique et à toute

11. Julia Kristeva a clairement défini ce transfert structural entre signification et énonciation. La signification sous-entend l'énonciation, la phrase, mais puisque la phrase se trouve pulvérisée, ou plutôt soumise à une autre logique, elle est complètement disjointe du code sous-jacent qui la définit comme telle (cf. J. Kristeva, La révolution du langage poétique, Paris, 1974 , p. 272 et suiv.).

12. Mais le poète qui a le plus lucidement analysé et évoqué cette transsubstantiation de la structure syntaxique a été sans doute Mallarmé : " l'œuvre pure implique la disparition élocutoire du poète, qui cède l'initiative aux mots, par le heurt de leur inégalité mobilisés : ils s'allument de reflets réciproques comme une virtuelle traînée de feux sur des pierreries, remplaçant la respiration perceptible en l'ancien souffle lyrique ou la direction personnelle enthousiaste de la phrase " (cf. S. Mallarmé, OEuvres Complètes, Paris, 1951, [La Pléiade], p. 366).

13. La voix peut être, par exemple, indépendante du langage pour - et chez -- les enfants en âge prélocutoire. Les animaux aussi, d'ailleurs, savent apprécier les différentes intonations de voix. 
contrainte distributionnelle. C'est que le langage poétique aurélien, et plus en général tout langage poétique, ne doit pas et ne peut pas être considéré comme une succession d'unités, de mots, entrant dans des paradigmes, c'est-à-dire "des ensembles des possibles sémantiques parmi lesquels un seul aurait été choisi et resterait par là même associé à ceux qui en ont été exclus " ${ }^{14}$. L'endroit où le mot figure dans la distribution linéaire (horizontale, mais aussi verticale), qu'impose la versification : mètre $\rightarrow$ rime $\rightarrow$ strophe(s) $\rightarrow$ refrain, décide de son signifié ${ }^{15}$, mais aussi des signifiés des autres unités figurant sur cette droite spatiale, rendue courbe par la droite temporelle de la scansion, véritable sonagramme de tout poème à forme fixe. Les notions d'environnement textuel, de commutation, de paradigme semblent alors imploser — même si des fragments de la structuration syntagmatique de la langue sont encore présents et perceptibles dans le texte poétique - sous les contraintes de la linéarité circulaire, et sous la polysémie structurellement active que crée la présence des tropes. C'est la chaîne normative même de la structuration du sens en sèmes qui est remise en question par les «infractions» poétiques, et cela quel que soit leur degré de compatibilité ou d'intégration avec le normal fonctionnement de la langue. Or, de toute évidence les termes mêmes d'infraction, d'implosion ou de rupture ne sont pas neutres, et dissimulent, derrière leur technicité prétendue, une primauté théorique ${ }^{16}$ de la structure syntaxique sur la phrase poétique, que les grammairiens ont depuis toujours cherché à théoriser, faute de pouvoir réellement la vérifier. Mais, dans une autre perspective, on peut les accepter,

14. Voir D. Maingueneau, Linguistique Française. Communication, Syntaxe, Poétique, Paris, 1992, p. 153.

15. Parfois aussi de son signifiant graphique et phonique. Il suffit de penser aux vers holorimes ou à certaines expériences de Robert Desnos. Chez Charles d'Orléans, ces calembours sont plus rares que chez les autres poètes de l'époque, mais néanmoins présents (voir $R$. CXXVIII 12 "J'an dy ce que mon cuer sent en ce ").

16. Il ne s'agit pas, évidemment, d'une suprématie innée, comme suffirait à le prouver l'histoire des littératures européennes, pour lesquelles la production orale, par définition mouvante, précède la production littéraire (en particulier en vers), celle-ci toujours en avance sur la production théorique : traités de versification ou de rhétorique, et surtout sur les efforts de normalisation des grammairiens. 
parce qu'ils mesurent aussi, et cela quel que soit leur connotation normative, la distance - ou peut-être faudrait-il dire avec J. Derrida la différance - qui sépare nécessairement un énoncé d'un texte poétique, la convention syntaxique du style ${ }^{17}$. L'impasse qui se cache derrière leur caractère classificatoire peut servir, en réalité, à nous faire prendre acte du fait que tous les systèmes grammaticaux ont désormais manifesté leur impuissance conceptuelle à saisir, décrire et ranger dans leurs graphes génératifs les noyaux sémantiques des mots et les mouvements suprasyntaxiques de la langue, qui sont pourtant à l'origine des figures de signification.

Chez Charles d'Orléans, les figures rhétoriques jouent justement cette fonction centrifuge qui sert à conférer au langage un dynamisme créateur asyntaxique. Le poète dessine sa propre organisation du langage, qui est, in primis, une transsubstantiation de sa pensée, fondée sur des mécanismes synésthésiques qui anticipent, parfois, sur ceux utilisés quatre siècles plus tard par certains poètes décadents ou surréalistes ${ }^{18}$. Mais la métaphore aurélienne apparaît comme entièrement lexicalisée, absorbée dans le tissu poétique, dérhétorisée, car la signification a dévoré les signes ${ }^{19}$, tous les signes. Le processus métaphorique ne fonctionne que rarement sur la comparaison qui explicite l'intersection sémique ${ }^{20}$, et cela en particulier dans les Rondeaux, où les codes rythmiques et rimiques, et la ronde des

17. Ils sont, en un mot, la preuve, plus encore que la conséquence, de la chasse au mystère de la poésie que grammairiens et linguistes mènent depuis Aristote, dans un esprit de systématisation systématiquement bafoué par les impertinences des poètes.

18. Je pense à certains poèmes de B. Cendrars, de J. Gracq ou de P. Éluard, mais aussi au J. Laforgue de Salomé : «[..] et ces plantations d'asperges confites et tuméfiées dans l'alcool du Silence " (cf. J. Laforgue, Moralités Légendaires, Salomé, Paris, 1977, p. 127). Sur l'esthétique de la décadence et son imagerie, on peut lire des pages éclairantes dans le livre de Pierre Jourde, L'Alcool du silence, Paris, 1994.

19. J'emprunte cette image à M. Merleau-Ponty, qui dans sa Phénoménologie de la perception, analyse de façon exemplaire le rapport de cogenèse que lie l'idée et la parole, la pensée et l'expression (cf. M. Merleau-Ponty, Phénoménologie de la perception, Paris, 1945, p. 213, et plus en général le chapitre VI, p. 203-232).

20. Ce terme désigne ici la partie commune à la mosaïque de deux sèmes soumis à un processus métaphorique. 
refrains, impliquent rupture syntaxique et rupture mélodique ${ }^{21}$ de la phrase poétique. Or, l'aspect le plus original du processus métaphorique aurélien est sans aucun doute celui de l'utilisation des métaphores in absentia, où l'intersection sémique n'est pas explicitée. et où il semblerait qu'il y ait une faible focalisation syntaxique du sème du lexème mis en valeur par le poète. Dans ces métaphores in absentia, le lexème se voit souvent affecté par un phénomène de virtualisation de sèmes que seul le contexte peut justifier ou valider. D'ailleurs, selon Jakobson «l'ambiguïté est une propriété intrinsèque, inaliénable, de tout message centré sur lui-même " ${ }^{22}$, en un mot un corollaire obligé de la poésie. Toutefois, explicitée ou non, l'intersection sémique, ou si l'on préfère la similarité entre les propriétés de deux sémèmes, est le fondement ${ }^{23}$ de tout procédé métaphorique, et cela quel que soit le mode de focalisation syntaxique. Or, il est précisément des séquences d'images chez notre poète, qui ne relèvent que partiellement de l'allégorie ${ }^{24}$ parce qu'elles supportent mal, ou ne supportent pas du tout, une lecture littérale («Forêt de Longue Actente", "Nef d'Esperance", "Livre de Pensee ", etc. ${ }^{25}$ ), et ne dévoilent que partiellement une vision du monde visible, qui serait signe du monde invisible. De surcroît, ces images ne sont presque jamais codifiées - sinon rarement par la tradition littéraire ${ }^{26}$ - et de ce fait difficilement reconnaissables

21. Voir P. Zumthor, Essai de Poétique Médiévale, Paris, 1972, p. 94.

22. Voir R. Jakobson. Essai de linguistique générale, Paris, 1963, p. 238, et plus généralement le chapitre XI.

23. Sur l'interprétation métaphorique comme mode de construction de la similarité, voir M. Black, Models and Metaphors, Ithaca, 1962, p. 37 : P. Ricour, La métaphore vive, Paris, 1975, p. 246; U. Eco, Les limites de l'interprétation, Paris, 1992, p. 156.

24. P. Zumthor et $\mathbf{A}$. Strubel préfèrent parler, dans ces cas, de réification. Mais la réification semble se fonder principalement sur une focalisation du sème due essentiellement à un transfert unidirectionnel sans aucune intersection donc … du sème du terme concret (verbe ou substantif) vers le terme abstrait (cf. A. Strubel, op. cit., p. 174-175).

25. Armand Strubel a relevé, utilisant son schéma de classification, 104 occurrences de ce type d'énoncés (cf. A. Strubel, op. cit., p. 183-185).

26. Dans le même article, A. Strubel a justement rappelé que l'on trouve dans la Psychomachia, mais aussi dans le Tournoiement Antechrist de Huon de Méry ou encore chez Jean de Meun, de nombreuses énumérations fondées 
comme allégoriques. D'autre part, la présence ambiguë de la particule DE, dans ces séquences d'images, semble instituer entre deux ou plus sémèmes, un rapport de subordination syntaxique, une relation de possession, de soumission ou de hiérarchie, qui semble rattacher ces figures davantage au mode de la personnification, ou de la personnification emblématique, selon la définition de Bloomfield (ou encore de la réification ${ }^{27}$ ), qu'à celui de la métaphore traditionnelle.

En réalité, il semblerait qu'il soit possible d'identifier dans cette particule DE une marque syntaxique de processus, c'est-à-dire de transition entre les composantes sémantiques du sémème concret et celles du sémème abstrait (l'exemple de «forest » et «Actente »). Elle instituerait alors plus une proportion qu'une similarité, parce que grâce à elle les deux lexèmes ne seraient pas remplacés mais posés in praesentia dans la manifestation linéaire du vers. Il y aurait, alors, une interaction métaphorique qui se produirait entre les deux contenus, ou mieux encore un transfert total des propriétés (feature transfer), et dans quelques cas même un transfert de catégories.

Ce transfert de contenus implique une absurdité du sens de la lettre, par conséquent une fausseté de l'encyclopédie, qui devrait être ressentie comme telle aussi bien par le poète que par son lecteur. Mais, alors que cette absurdité déclencherait logiquement de la part

sur le paradigme : nom concret - DE - nom abstrait, qui pourraient avoir servi comme modèle à Charles d'Orléans, et qui fonctionneraient, donc, comme référent intertextuel. Toutefois, le cadre thématique de ces énumérations ect celui stéréotypé de l'armement du combattant des armées de Vice et de Forıune, qui ne figure pas dans le corpus du Duc (Ibid., p. 177-180).

27. On pourrait aussi introduire la notion d'allégorèse, déjà proposée par P. Zumthor à propos de la combinaison de métaphores et personnifications (cf. P. Zumthor, Langue, texte, énigme, Paris, 1975, p. 105); toutefois sur le plan littéral, celui de l'expression, le rôle de sujet semble, chez Charles d'Orléans, être joué davantage par des réifications, qui dynamisent sémantiquement moins l'action métaphorique, et alimentent moins ou marginalement le développement allégorique du poème. D'autre part, son champ d'utilisation serait ambigu, car le terme a aussi été employé pour désigner toute une tradition exégétique antérieure au Moyen Âge (cf. A. Strubel, La littérature allégorique. Limites et marges de la pratique allégorique, dans Précis de Litlérature francaise du Moyen Age, sous la dir. de Daniel Poirion, Paris, 1983, p. 249). 
du récepteur la réaction interprétative métaphorique devant un énoncé autrement perçu comme anormal, elle devrait, au contraire, paralyser l'intention du locuteur qui, l'utilisant, risquerait, d'une part, de ne pas "voir" prendre au sérieux son message, et, d'autre part, renoncerait à la validité qu'attribue à l'énoncé sa valeur référentielle. En d'autres mots, ce nouveau monde du locuteur, cet ailleurs verbal, où forêt n'est plus la forêt de l'encyclopédie, parce que sa valeur dénotative, son référant, a été transférée dans "Longue Actente », qui lui a cédé le sien (et inversement), ne peut pas être un monde possible, où le locuteur projetterait le contenu du sens littéral, puisqu'une expression métaphorique ne peut aucunement prendre l'aspect d'un contre-factuel. Aussi parce que le récepteur pourrait être tenté de croire que l'anomalie est dans l'émetteur plutôt que dans l'énoncé, et renoncer à tout effort de décodage. Si une telle volonté hermétique de la part du locuteur ne peut pas être totalement exclue - il suffirait d'imaginer sous le fard courtois de notre prince grimacer un nouveau Sisyphe, heureux de brouiller la transmission du sens que son énoncé est censé porter, entraînant ainsi dans son cercle infernal le lecteur-pécheur; ou, plus simplement, certains exemples d'écriture automatique -, elle paraît improbable, sinon absurde, dans un contexte poétique soumis aux contraintes de la rime, du rythme, du poème à formes fixes, par définition, donc, codifié et impressif, et symbole même de recherche de la communicabilité.

En réalité, il semble bien que les risques encourus par le poète soient parfaitement calculés, et même nécessaires à l'intelligibilité globale de sa pensée, qui se fonderait, ainsi, sur l'opacité du texte. Son choix apparemment hermétique peut très bien être expliqué avec ce que $\mathrm{M}$. Black appelle la capacité constitutive de la métaphore : «Certaines métaphores nous rendent capables de voir des aspects de la réalité que la production même de métaphores aide à constituer. Mais il n'y a là rien d'étonnant si l'on songe que le monde est sûrement le monde sous une certaine description et un monde vu d'après une certaine perspective. Il est des métaphores pouvant créer une certaine perspective " ${ }^{28}$. C'est-à-dire un mode de production qui se servirait du symbole et de son langage, de l'image, pour révéler ce

28. Cf. M. Black, More about Metaphore, dans Metaphor and Thought, par A. Ortony, Cambridge, 1979, p. 39-40. 
qu'il semble cacher. C'est, d'ailleurs, ce que pense aussi Daniel Poirion, quand il parle d'une poésie aurélienne qui «donne à voir pour contraindre à penser ${ }^{29}$ : à réfléchir sur un sens qui se dérobe pour mieux se structurer à travers, d'une part la recherche du style, et d'autre part la quête interprétative ${ }^{30}$ : la syntaxe de la phrase poétique se posant, alors, comme la direction linéaire empruntée par la recherche bijective de son sens.

Cependant, pour qu'il y ait création de perspectives, par conséquent, quête du sens, il faut naturellement que ces images n'appartiennent pas, ou pas encore, au savoir encyclopédique (médiéval, mais aussi uchronique); qu'elles soient, donc, créées pour rendre compte d'une expérience - ou de plusieurs - intérieure, et inscrites dans un contexte qui leur sert de tain ; qu'elles soient, en un mot, un texte déjà donné. En effet, ces métaphores créatives, selon $\mathrm{S}$. Briosi ${ }^{31}$, naissent d'un choc perceptif, qui permet au locuteur de se mettre en rapport avec le monde qui précède le travail linguistique, dont celui-ci est la conséquence. Certes, U. Eco a, sans doute, raison de croire qu'"il semble difficile de dire si l'auteur a eu d'abord une expérience psychologique qu'il a ensuite traduite en langage, ou s'il a eu d'abord une expérience linguistique dont il a tiré ensuite une disposition différente pour voir le monde " ${ }^{32}$ - encore qu'on puisse difficilement concevoir un processus linguistique qui puisse inférer sans être inféré. En revanche, il a sans doute tort de penser que «le monde intérieur de l'auteur [...] est une construction de l'acte d'interprétation métaphorique, non une réalité psychologique [...] qui motive l'interprétation elle-même " ${ }^{33}$. Autant dire que les états d'âme sous-jacents, par exemple, aux images de la "Forest de Longue Actente » ou de la « Nef d'Esperance ", n'en sont pas, et que leur perception n'existe que parce que l'intention du lecteur (intentio lectoris) les provoque en les actualisant; que le monde intérieur de Charles d'Orléans, donc, est en réalité un reflet en débris de celui du

29. Cf. D. Poirion, La Nef d'Esperance. Symbole et allégorie chez Charles d'Orléans, dans Mélanges Jean Frappier, Genève, 1970, t. II, p. 928.

30. Dans une dimension eschatologico-littéraire, la métaphore créative est métaphore du plaisir du texte, imago aussi bien de l'intentio auctoris que de l'intentio operis, cadre culturel obligé de l'intentio lectoris.

31. Cf. S. Briosi, Il senso della metafora, Roma, 1985.

32. Cf. U. Eco, op. cit., p. 162.

33. Ibid., p. 162. 
lecteur; ou encore que les métaphores créatives qui constituent les symboles de sa différance, ne sont pas un mode d'expression ou de communication, mais uniquement un mo(n)de de perception où le poète disperserait ( damnerait»?), dans le transfert entre le concept et les mots, l'appréhension de son moi ; en somme, que l'activité du poète, sa quête du moi, ne parviendrait jamais à produire ou plutôt faudrait-il dire à reproduire -.- l'image, la figure de son monde intérieur consciemment ou inconsciemment alors que la "simple " activité de l'interprétation métaphorique (ou de la lecture) réussirait à refléter ce monde que le poète serait le seul, alors, à n'avoir pu et su (se) dire. Mais, alors, quelle conscience de soi et de l'acte poétique inspirait et motivait Charles d'Orléans lors de son aveu du pénible métier d'écriture : "C'est privé martyre / Plus penser que dire »! Pour quoi, pour qui, quelques siècles plus tard, Flaubert consommait-il sa voix dans ce gueuloir simulacre de son style? Et, surtout, pourquoi arrêtait-il ? Sans compter que la liberté d'action de l'intentio lectoris aussi n'est qu'apparente, puisque sa prétendue souveraineté serait de toute façon limitée par les bornes du savoir encyclopédique, et de facto niée.

Il ne s'agit évidemment pas de nier le rôle joué par l'intentio lectoris dans le procédé de décodage des métaphores auréliennes (ou autres) ; ce serait, de plus, une tentative qui se transformerait en une inférence de l'inverse ${ }^{34}$. Cependant, celle-ci ne peut pas ne pas supposer une intention de l'auteur qui se traduit dans le texte, et que le savoir encyclopédique du lecteur ne sait, dans la meilleure des hypothèses, qu'éclairer par contraste, aussi bien par son acceptation que par son refus. D'autant plus qu'on peut très bien supposer un texte sans autre lecteur que son auteur, degré zéro de l'intentio lectoris - c'est probablement le cas, parfois, aussi de notre Duc dans certaines chansons et ballades écrites durant sa captivité anglaise...., ou plus simplement un texte au stade de brouillon, qui continue pourtant de posséder une intentio operis et évidemment d'être soumis

34. Tout décodage demande un nouvel encodage, mais il est de toute évidence qu'entre un décodage-encodage purement conceptuel, ce qui est le cas chez la plupart des récepteurs, et un décodage-encodage explicité, textuel, il y a toute la distance qui sépare le concept de la parole, ou, pour reprendre la formule de M. Merleau-Ponty, la parole parlante de la parole parlée, et en définitive l'intentio operis de l'intentio lectoris. 
à l'intentio auctoris. Plus, quinze ans de critique génétique ont montré qu'un tel texte, même soumis à une activité de lecture exogène, semble ne pas activer les mécanismes de l'interprétation, mais plutôt ceux de l'enquête philologique, voire de la glose biographique. Il semblerait, en effet, que pour se déclencher, l'intentio lectoris nécessiterait la présence d'une volonté esthétique explicite de l'auteur et du texte, comme reconnaissant la nécessité de l'impulsion d'une intentio auctoris non seulement autographe mais aussi authentique, qui seule autoriserait alors la nécessité ou le plaisir de l'errance interprétative.

Je voudrais montrer, alors, que dans le cas de certaines séquences d'images utilisées par Charles d'Orléans, et plus en général dans ce genre de séquences créatives, la métaphore dépend presque exclusivement de la sentence meaning dont parle J. Searle, plutôt que du reader's meaning ${ }^{35}$, qui n'en est que la substance de contraste. Je tâcherai de mettre en évidence qu'en réalité le savoir encyclopédique de l'homme médiéval - tout comme celui d'un récepteur moderne qui n'aurait pas encore intégré le texte aurélien - n'interagit que dans une faible mesure avec le texte, et que si l'on veut tenter d'avoir une interprétation qui ne soit ni illégitime, ni antiéconomique (celle, par exemple, que l'on jugerait comme convenable et acceptable de la part d'un étudiant lors d'un examen), on doit suivre certaines directions qui sont inscrites dans le texte, exactement comme il nous arrive de le faire dans l'interprétation - ou pour mieux dire dans la reconnaissance - des termes syncatégorématiques. Ces directions obligent, en effet, le lecteur à narcotiser la plupart des propriétés de l'énoncé métaphorique, et plus exactement toutes les propriétés périphériques des sèmes, résultant d'une stratification connotative encyclopédique, et à activer, ou plutôt à reconnaître comme active la propriété sémantique première, ce noyau correspondant à la propriété dénotative innée ${ }^{36}$. Ces séquences se révèlent alors comme des structures sémiques transparentes, pures, qui ne supportent aucune autre activité métaphorique que celle véhiculée par le "mouvement sémantique qui court comme une eau vive sous la

35. Cf. J. Searle, Sens et expression : études de théories des actes de langage' (trad. franç.), Paris, 1982.

36. Celle qui lie à l'origine signifiant et signifié. 
glace " ${ }^{37}$, c'est-à-dire celle construite par et pour le monde intérieur du poète, et que le lecteur ne peut que suivre au gré des courants : épaves de conscience et pensées qui refluent vers le moi du poète. Le seul savoir encyclopédique que le récepteur peut donc utiliser est celui correspondant au sens littéral de l'énoncé (en somme un savoir encyclopédique au degré zéro, autrement dit un savoir sémantique), parce que le système d'interprétation métaphorique de ce sens ne lui est fourni que par le texte, tout le texte aurélien - ce qui prouve, aussi, au passage, l'indissolubilité énonciative, donc narrative, de son cuvre : Ballades et Rondeaux , constituant, ainsi, un savoir que l'on pourrait définir comme textuel (ou à la rigueur monographique !), et qui trouve dans le monde intérieur du poète, qu'il soit reflet ou construction de son expérience humaine, la seule légitimité possible, ou du moins la seule qui procure le plaisir du texte. Ces métaphores ne fonctionnent, donc, que dans un univers culturel et textuel donné, dont elles sont une figure. D'ailleurs, une même métaphore n'a pas toujours le même sens, malgré le fait que le savoir encyclopédique de l'interprétant soit une invariance. Prenons, par exemple, le cas, qui est loin d'être exceptionnel, de la «Forest de Longue Actente ", véritable locus amoenus de la poésie aurélienne, mais non seulement ${ }^{38}$. Le duc utilise cette image une fois dans les Ballades et deux fois dans les Rondeaux. Il la propose, ensuite, comme motif poétique à sa cour. Interviennent, alors, dans le débat, huit correspondants poétiques du cercle de Blois, avec des variations sur le thème ${ }^{39}$. Plus ou moins à la même époque (1456-1457), un

37. Cf. D. Poirion, Le lexique de Charles d'Orléans dans les Ballades, op. cit., p. 31 .

38. L'image de la forêt, lieu dont on ne sort qu'à grand-peine, est un des topoi de la tradition patristique et médiévale, que l'on retrouve dans toute la littérature européenne du $\mathrm{XIV}^{\mathrm{e}}$ et $\mathrm{XV}^{\mathrm{e}}$ siècles. Il suffit de penser, à ce propos, à "la selva oscura " dans laquelle se retrouve Dante au début de la Divine Comédie. On peut parler même de résurgence, parce qu'on trouve la même séquence, un siècle plus tôt, en 1369, dans l'Espinette Amoureuse de Jean Froissart (v. 2090), et, plus près du Duc, en 1424, dans La Belle Dame sans mercy d'Alain Chartier (Alain Chartier, La Belle Dame sans mercy. Copies des Lettres, par A. Piaget, Genève, 1949, p. 35).

39. Le Duc utilise cette image dans la Ballade CV 1, et dans les Rondeaux CCXXV 1-9-15 et CCXXVIII 1-9-15. Elle est ensuite reprise par Jacques bastard de la Tremoille dans la Ballade CIX ${ }^{a}$ 8-16-24-28. Sept autres poètes 
autre prince-poète, cher ami de Charles d'Orléans, le roi René d'Anjou, en fait un des loci amoeni de son Livre du cuer d'amours espris ${ }^{40}$, véritable arsenal d'images et motifs allégoriques, souvent utilisés au second degré. Or, ce même syntagme minimal est soumis chez René d'Anjou, de même que chez les correspondants poétiques du cercle de Blois, aussi bien au principe de contextualité qu'à celui de l'intertextualité, et on pourrait facilement montrer que dans ces cas les interprétations métaphoriques autorisées par le contexte sont toutes entières contenues dans le référent intertextuel, c'est-à-dire l'encyclopédie. Autrement dit, ces métaphores ne demandent aucun effort interprétatif nouveau, ou du moins authentique, mais tout simplement activation des propriétés connotatives de leurs constituants sémantiques ou de ceux de l'environnement textuel et surtout paratextuel. Elles invitent à voyager dans un espace et dans un lieu convenu : celui dangereux de la silva-labyrinthe, dont on ne sort qu'à grand-peine, et en même temps, à travers la marque catégorielle du temps, véhiculée par le syntagme Longue Actente, suggère par analogie l'autre voyage, celui de la vie, de toute vie. Le contexte n'interfère que dans une moindre mesure, avec leur message, et le leur, plus qu'un phénomène lexical, semble être un phénomène culturel, d'où toute invention créative, toute marque de subjectivité, aussi bien active que passive, d'ailleurs, sont bannies, ou du moins banalisécs, dans un simple excrcicc interprétatif à différenticl culturel, qui est aussi noble et peut sécréter ses délices, mais qui n'explique pas nécessairement tous les mécanismes de l'acte poétique. Mais si à l'évidence ces forêts se révèlent comme pure convention élocutoire, thème poétique, topos métaphorique et interprétatif, en quoi et pourquoi la silva aurélienne serait-elle innovatrice et non para-

du cercle de Blois ont aussi apporté leur contribution au débat sous forme de rondeaux, selon le principe de la aequalitas numerosa, cher à la rhétorique médiévale : $R$. CXXXI 1-9-15 (Philippe Pot), $R$. CXXXII 1-9-15 (Antoine de Lussay), $R$. CXXXIII 1-9-15 (Guiot Pot), $R$. CXXXIV 1-9-15 (Gilles des Ormes), $R$. CXXXVII 1-9-15 (Jacques bastard de la Tremoille), $R$. CCXXVI 1-9-15 (Madame d'Orléans) et $R$. CCXXVII 1-9-17 (Fredet). La numérotation des poèmes fait référence à l'édition de Pierre Champion, Charles d'Orléans. Poésies, 2 vol., Paris, 1923-27.

40. Cf. Le livre du Cuer d'amours espris, par S. Wharton, Paris, 1980 [10/18], p. 33 : «En la forest de Longue Actente/Tu entreras selon m'entente $"$. 
phrasable? Nous avons déjà souligné le rôle marginal joué par l'encyclopédie dans le décodage de cette métaphore aurélienne, il est intéressant alors de vérifier à travers la comparaison de deux rondeaux joués sur ce même thème, le premier du prince, le second de son épouse, le degré d'intégration formelle et de contenu de la métaphore, et la capacité d’activation des propriétés sémantiques dénotatives et connotatives que présentent les deux contextes.

\section{R. CCXXV (Charles d'Orléans)}

En la forest de Longue Actente.

Par vent de Fortune Dolente,

Tant y voi abatu de bois

Que sur ma foy, je n'y cognois

A present ne voye, ne sente.

Pieça, y pris joyeuse rente, Jeunesse la payoit contente, Or n'y ay qui vaille une nois,

En la forest de Longue Actente.

Vieillesse dit, qui me tourmente : Pour toy ny a pesson, ne vente, Comme tu as eu autresfois ;

Passez sont tes jours, ans et mois :

Souffize toy et te contente,

En la forest de Longue Actente.

\section{R. CCXXVI (Madame d'Orléans)}

En la forest de Longue Actente.

Entree suis en une sente,

Dont oster je ne puis mon cueur :

Pour quoy je viz en grant langueur

Par Fortune qui me tourmente.

Souvent Espoir chascun contente,

Excepté moi, povre dolente,

Qui, nuyt et jour, suis en doleur

En la forest de Longue Actente.

Ay je donc tort se me garmente Plus que nulle qui soit vivente? 
Par Dieu, nennyl, veu mon maleur; Car, ainsi m'aist mon Createur Qu'il n'est paine que je ne sente En la forest de Longue Actente.

Le cadre allégorique semble être le même dans les deux cas, celui déjà évoqué de la silva périlleuse, figure du pénible voyage de la vie, où l'homme et la femme se retrouvent avec des compagnons courtois traditionnels : Fortune, Espoir, Jeunesse-Vieillesse - bien que ces deux dernières personnifications n'apparaissent pas, et pour cause, dans le poème de Madame d'Orléans. Le procédé allégorique fonctionnerait, donc, comme convention élocutoire, les deux poètes prêtant à leurs lecteurs une familiarité avec cet univers du symbole qui rend superflue toute explication. C'est que le lecteur médiéval, en effet, est désormais habitué à évoluer et à s'orienter dans un univers de signes autoréférentiel, véhiculant, donc, un message, ou plutôt l'image d'un monde, immuable, et de ce fait parfaitement transparent et intelligible dans son apparente opacité. L'allégorie n'est autre, alors, qu'un clin d'œil allusif à un mystère qui n'en est pas un, et son caractère subjectif s'estompe jusqu'à disparaître dans l'objectivité d'un référent qui n'est plus sémantique mais culturel. Or, cette impression de spécularité, sinon de similarité, qui se dégage des deux poèmes, est due surtout à une erreur de perspective critique, à une extrapolation injustifiée et trompeuse d'un texte, celui de Charles d'Orléans, qui est strictement enchevêtré dans son contexte poétique, dont il n'est qu'une maille narrative, fût-ce celle qui dévoile, selon l'expression de Montale, "la formula che mondi possa aprirti». Tous les mots de ce texte proposent ou suggèrent des renvois, explicites ou non, à des situations psychologiques, à des états d'âme, à des objets ou tout simplement à des sonorités, que l'on retrouve dans le chaos apparent de la création éparpillés dans les autres poèmes du Duc ${ }^{41}$, et qui construisent tous ensemble un monde

41. Trois travaux, en particulier, analysent, même si de façon indirecte, le système complexe de références sémantiques, phonétiques, rimiques qui parcourent tout le texte aurélien, à commencer par ce rondeau (cf. D. Poirion, Le lexique de Charles d'Orléans dans les "Ballades", op. cit. : A. Planche, Charles d'Orléans, ou la recherche d'un langage. Paris, 1975; C. Galderisi, Le lexique de Charles d'Orléans dans les Rondeaux, op. cit. Il suffira ici de rappeler, outre évidemment les autres emplois de l'image de la «forest 
poétique cohérent dans sa diversité, parfois dans sa duplicité, avec une architecture narrative du vers, de la strophe et des refrains dense et transparente à la fois, où tout se tient merveilleusement en équilibre par la magie du rythme et du style. Un réseau complexe qui se fonde d'abord sur la réalité, non seulement celle qui résulte de l'observation biographique, mais celle construite à travers la recherche des combinaisons de mots qui mettent en valeur le rôle originel des sèmes, et où des références apparemment abstraites, comme «Fortune Dolente», ou concrètes comme « pesson» ou « rente», contribuent en réalité à étoffer la réflexion à travers l'infiltration mesurée de l'hic et nunc, tout en la parant d'un voile de pudeur et de légèreté. Les sèmes des mots concrets se greffent alors sur ceux des abstracta comme sur autant de clichés immaculés, les transformant en stylèmes, en émotion, en poésie. Cette «Forest de Longue Actente " se révèle alors à travers la lecture de tous les sentiers qu'y mènent ballades, chansons et surtout rondeaux, comme à la fois lieu de l'émotion et état d'âme chosifié, figure de la vie de Charles d'Orléans : la vie de l'homme et celle du poète. Ce qui explique aussi pourquoi, à la différence de la « selva oscura", le poète ne cherche pas à s'évader de la "Forest de Longue Actente ", et accepte aussi bien la «joyeuse rente» de Jeunesse que les tourments de Vieillesse. Parce que sentiment poétique et mémoire, joie de vivre et souvenir

de Longue Actente ") que les deux figures sinistres de Jeunesse-Vieillesse, qui veillent à l'orée de cette prison-forêt, et qui par leur présence actualisent sémantiquement le sème de Longue Actente, mettant en valeur sa linéarité temporelle immuable, donc dramatiquement cyclique et universelle dans son infinité, sont les géôliers-compagnons d'un voyage autour du rondeau qui va durer jusqu'à la mort, ou du moins jusqu'à la fin de la production aurélienne, et qui les verra apparaître dans les Rondeaux presque toujours ensemble (onze fois sur douze Jeunesse est en compagnie de Vieillesse). Mais là aussi, et une fois de plus, un lecteur qui, se fiant à son savoir encyclopédique, chercherait à interpréter cette sorte de Janus bifrons à travers les schémas de la tradition allégorique courtoise, serait fort déçu de constater que pas une seule fois ces personnifications n'ont été décrites physiquement par le poète, qui en a fait plutôt que des symboles du temps qui bat la mesure de son existence, les parques-hamadryades de cette forêt de Longue Attente, dimension de l'esprit où "le poète peut imaginer une existence autre que celle que le destin lui a réservée, et où les arrêts de Fortune seraient cassés ou décriés par une dame nommée Poésie" (Charles d'Orléans. L'Écolier de Mélancolie, par Claudio Galderisi, Paris, 1995, p. 8). 
des désillusions, conscience de l'authenticité mais aussi de l'universalité de son destin se condensent et s'incarnent, comme déjà avec l'image du "Livre de Pensee", dans un hologramme poétique et affectif qui est une miniature-miroir pathétique de son esprit. La métaphore offre, donc, dans la richesse des secrets qu'elle laisse filtrer non pas la clé de son interprétation, mais un instantané des nombreux fils poétiques qui se noucnt au travers d'clle, et qui tous participent et témoignent de l'intentio auctoris et de l'intentio operis. Des fils subtils, que l'on peut suivre au gré de la lecture et des égarements du poète, si seulement le lecteur parvient à se défaire de son lest encyclopédique, de son expérience linguistique. C'est alors que les mots dépouillés du fard des circonstances et de la fange de l'usage quotidien retrouvent dans l'élégance et dans la musicalité de la phrase poétique la netteté de leur signe originel, l'éclat du sème à l'état pur, où toutes les intentiones se fondent dans leur recherche, dans leur nécessité mutuelle, tous, poète et lecteurs, recherchant, en fait, un lieu de rencontre dans l'inutile poésie.

À côté de ce "privé martyre » qui se consume derrière cette apparente facilité, nous avons, d'autre part, un texte, le rondeau de Madame d'Orléans, qui, tout comme ceux des autres sept correspondants poétiques de Charles d'Orléans, nous présente un simple exercice de style, parfaitement interprétable à travers le schéma culturel de la convention, l'encyclopédie. Ici, l'image de la « Forest de Longue Actente " non seulement ne semble figurer aucune expérience psychologique antérieure, mais elle ne préfigure non plus aucune réflexion sur une situation ou un état d'âme qu'elle contribuerait à comprendre, à saisir ou à rendre perceptible. Elle semble gratuite aussi bien sur le plan du contenu que sur celui de l'expression. D'une part la présence du substantif de lieu forest n'est justifiée que par l'écho sémantique de sente ("sentier»), et n'atteint même pas le statut de locus amoenus, forest pouvant être, en effet, parfaitement remplacé par un autre substantif de lieu (Château. Prison, etc.); d'autre part la marque temporelle véhiculée par Longue Actente n'est validée par aucune autre reprise sémantique interne au rondeau, et pourrait aussi être commutée avec un quelconque syntagme nominal (Douloureuse Souffrance, Pénible Vie, etc.) sans que l'équilibre rhétorique et l'intelligibilité du rondeau en souffrent. Elle est le cadre rhétorique d'un voyage artificiel qui ne dure que l'espace d'un vers, ou d'une strophe, tout au plus. 
Mais la raison principale de ce sentiment de passe-temps sans conséquences que l'on éprouve à la lecture de ce rondeau de Marie de Clèves ne réside pas dans l'échec formel, dans une manière rhétorique peu ou mal maîtrisée, dans un allégorisme seulement partiellement réussi, mais dans l'absence de souffle poétique, dans le caractère superficiel et scolastique d'une image qui ne cache, et donc ne dévoile, que son moule rhétorique, ou tout au plus son référent culturel. Quelle expérience poétique a-t-elle vécu dans sa "forest de Longue Actente", cette "povre dolente"? et quelle est cette "Longue Actente", dans laquelle elle serait entrée, et surtout que signifie-t-elle? Un lecteur malin, qui connaît le mauvais rire du roi Louis XI sur les naissances qui vinrent animer le foyer du Duc sexagénaire, pourrait s'amuser à y voir, par exemple, une allusion à la longue expérience de mariage que la jeune Marie de Clèves devait endurer pendant vingt-cinq ans avec le vieux Charles d'Orléans ${ }^{+2}$, et de là élaborer autour de cette métaphore-allégorie une concaténation de miroirs psychologiques qui nous renverraient une image nouvelle et authentique d'une jeune femme-poète secrète et mystérieuse ; mais un tel exercice serait, outre que gratuit, parfaitement vain parce que les quelques vers que la princesse nous a laissés n'offrent aucun appui possible à une telle interprétation, et surtout n'expriment aucune tension psychologique, aucun état d'âme qui se traduise en émotion, en poésie, qui communiquent au lecteur, en la lui faisant partager, une telle vérité poétique. Qu'elle ait souffert ou pas dans sa vie à côté du prince, Marie de Clèves n'a pas fait de cette hypothétique souffrance un élément ou une raison de réflexion poétique, qui puisse aujourd'hui autoriser une telle lecture de sa "forest de Longue Actente".

La métaphore, donc, qui dans le texte du prince dégage une charge émotionnelle et créatrice première, une densité poétique vitale, fondée sur le caractère primitif et évocateur de sa valeur dénotative, est ici complètement éteinte, son sème originel dilué

42. Quand à sa délivrance, en 1440 , le prince âgé de quarante-huit ans, et vieilli par les vingt-cinq ans de captivité, épousa Marie de Clèves, celle-ci n'avait en effet que quatorze ans. En fait, il semblerait que toutes les métaphores créatives réussies se fondent sur un terme concret dont le caractère hautement symbolique (forêt, nef, livre) tient davantage des expériences vécues associées à leur schéma conceptuel que des propriétés dénotatives de leur sème. 
dans la convention, dispersé par le plaisant vent du jeu rhétorique, du divertissement courtois fin en soi; son isolement sémantique, l'absence de toute interaction contextuelle en font un hapax conceptuel, un déchet rhétorique que seul ses propriétés connotatives peuvent rendre intelligible. Mais elle ne nous apporte aucune connaissance, fût-elle celle d'une seizième joie de mariage, provoquant, au mieux, le plaisir d'une reconnaissance encyclopédique.

Toutefois, chez Charles d'Orléans, aussi, ces séquences d'images, ces métaphores emblématiques ou créatives, qui mettent en relation le monde des concrets et celui des abstraits, ne semblent pas toutes posséder la même intensité et la même force introspective, comme nous l'avons déjà vérifié chez Madame d'Orléans; parfois la "pollution" connotative, autrement dit la perception d'un schéma rhétorique traditionnel ${ }^{43}$, semble les affaiblir, les banaliser, en les assimilant à l'encyclopédie des interprétants et à la dimension spatiale de la syntaxe; d'autres fois, et c'est le cas le plus fréquent, leur immobilité sémantique semble être moulée sur un immobilisme visuel qui n'est autre que le miroir d'un vide perceptif : la conséquence d'une irroration insuffisante ou intermittente du sentiment poétique ${ }^{44}$. Souvent, ces images un peu baroques (cf. Ball. XXXII 6-8, le cœur enveloppé dans un "cuevrechief de plaisance/Et enclos, pour plus grant seurté, / Ou coffre de ma souvenance "), sont aussi reconnaissables parce qu'inscrites dans un contexte déjà métaphorique - nous avons aussi bien des métaphores filées que des allégorismes - et elle échouent poétiquement, car l'interprétation métaphorique provoquée par la reconnaissance de l'absurdité du sens littéral ne fait apparaître autre chose que ce même

43. Je pense, par exemple, au "roman de plaisant penser" (Ball. VIII), où à la « roche d'esperance » (Ball. L), ou encore à la «cité de Destinee " (Ball. $\mathrm{CV}$ ).

44. C'est le cas de ces véritables exercices de style que sont le $R$. CCLXXXV (" molin de pensee ", "farine de doulceur ", " son de destinee ", " eau de pleur, de joye ou de douleur»), et le $R$. CXIX ("fleur de souvenir", " erbe de loin de son desir", "poire d'angoisse", etc.). Ailleurs, parmi les rondeaux du Duc, on peut lire un poème de Benoist Damien, farci de formules de ce genre, où la gratuité et l'inconsistance de l'association substantif concret-substantif abstrait touche au ridicule, plus encore qu'au maniérisme (voir $R$. CCLXXIX 12 «bescuit durcy de langueur»). 
sens littéral, c'est-à-dire qu'elle dévoile un monde sémiotiquement absurde ou comique ${ }^{45}$.

Une telle prolifération de séquences de ce type, et avec des résultats aussi divers, doit nous inciter à réfléchir sur le rôle et la fonction du sémème concret et du sémème abstrait à l'intérieur du syntagme métaphorique, en essayant, évidemment, de ne pas tomber dans le piège d'une définition syntaxique du degré de cohésion métaphorique d'une séquence, qui serait en contradiction avec le concept d'interaction sémantique contextuelle, déjà évoqué à propos des multiples exemples de "Forest de Longue Actente" ${ }^{46}$. Un classement par champs d'action sémantiques de ces séquences permettrait d'élaborer un recoupement plus précis, et par conséquent une meilleure compréhension des mécanismes opérant lors de leur production.

Deux types principaux de combinaisons sémème concret-sémème abstrait semblent émerger, alors, à travers un tel découpage. C'est à partir du degré d'intégration conceptuelle, de coalescence métaphorique, que l'on pourrait tenter d'établir, plus encore qu'une politique, une poétique éditoriale des termes à majuscule qui soit réellement fondée sur leur mode de production rhétorique.

Nous avons une première catégorie de séquences, où l'on trouve associés à des abstracta récurrents chez le Duc, des noms concrets qui renvoient à une action (« aumosne ", " ambusche », " voyage », etc.), à un métier (« marchants »), à un manufacturé, à un élément naturel ou physiologique ( avirons", "cuevrechief», « eaue », « erbe ", " larmes", etc.), pour lesquels la particule DE semble bien instituer un rapport d'agent, de consistance, de matière. Dans ces cas ${ }^{47}$, il semble bien que les composantes sémantiques du sémème

45. En fait, il semblerait que toutes les métaphores créatives réussies fondent leur transfert sur un terme concret dont le caractère hautement symbolique (forêt, nef, livre) tient davantage aux expériences vécues associées à leur schéma conceptuel qu'aux propriétés dénotatives de leur sème. Ce texte déjà donné rendant. de fait, inutile tout effort herméneutique.

46. Si on essayait, en effet, de considérer ces séquences, d'un point de vue purement linguistique, comme des paralexèmes poétiques, et comme tels de leur appliquer les tests d'insertion, de commutation ou de coordination, pour vérifier leur degré de coalescence, on ne pourrait constater que leur absurdité, ou la parfaite extranéité sémantique des constituants.

47. Ce corpus se composerait alors des séquences suivantes:a) Ballades: 
concret ne parviennent pas à se greffer complètement sur celles du sémème abstrait, finissant même par estomper le caractère mythique de celui-ci, affaiblissant ou banalisant sa charge subjective. Aucune interaction métaphorique ne s'établit entre les deux contenus, moins

XIV 19 « la nue de tristesse », 21 « le beau temps de lyesse»; XXIII 19-20 «l'aumosne de partir / De lyesse"; XXVII 7 «la mer de fortune»; XXXII 6 "En ung cuevrechief de plaisance», 8-16-24-28 «ou coffre de ma souvenance ", 11 «En larmes de piteux penser », 14 " au feu d'esperance », 21 «Soubz clef de bonne voulonté"; XLIII 32 «L'habit de desconfort"; L 5 "sur roche d'esperance»; LXV 24-25 «l'estincelle / D'ardant desir»; LXVI 6 «du somme de soussi", 8-16-24-28 "Sur le dur lit d'ennuyeuse pensee»; LXIX 5 "Mains sierges de soupirs piteux»; LXXIII 1 "L'emplastre de nonchaloir»; LXXX 3 «l'arbre de plaisance», 10 «l'essor de largesse ", 18 "l'eaue de lyesse », 20 " du moisy de tristesse" ; LXXXI 29-30 " es fers te garderons / De soussi et pensement "; XCVIII 13 «De confort la voille ", 18 "L'eaue de fortune ", 20 "avirons d'espoir", 27 "vagues de tourment "; CII 17-18 «de consolacion / Quierent l'aumosne»; CV 4 «Ou voyage de desireirs"; CVI 13-14 «de l'endormye beuvra / De nonchaloir »; CVIII 1 " harnoys rouillé de nonchaloir», 2 «monture foulee de foiblesse", 4 «aux montres de lyesse»; CXI 8-16-24-28 «le vent de merencolie»; CXVII 7 "des verges de soussy"; CXXI 8 «de noir de tristesse"; b) Chansons: LVII 1-7-13 «ce soleil de plaisance»; c) Rondeaux : LXVII 5-6 "en frontirere/De dangereux parler»; LXXII 6 "Chemin de plaisant vent "; LXXX 1-7-13 «des arreraiges de plaisance»; LXXXI 10 «a cros de bonne esperance"; LXXXVI 2-8 "pouldre de plaisir ", 3 "le vent d'amoureux désir»; CXVIII 9 "Sucres de pensers savoureux"; CXIX 2 "la fleur de souvenir ", 6 «l'erbe de loing de son desir», 7 " poire d'angoisse ", 10 "Pouldre de plains", 11 "Feuille d'aultre", 12 « racine de jalousie"; CCXXV 2 «le vent de fortune dolente»; CCXXXI 2-8 «l'aumosne de doulceur»; CCLI 2-10-17 " des fievres de merancolie»; CCLXXX 2-3-10-11 «la tourmente / De deuil», 3-11 « le doulx temps d'espoir», 12 «marchans de longue actente»; CCLXXXV 1-7-13 "L'eaue de pleur, de joie ou de douleur », 5 «farine de doulceur », 6 « son de dure destinee »; CCCVII 2-8 "le vent de merencolie»; CCCXXIV 2-8 "Ou parfont tresor de pensee": CCCXXV 2-8 "l'eaue d'espoir "; CCCXXXII 8 "vent de desplaisance ", 14 "la marchandise d'esperance "; CCCCII 3 "L'ambusche de plaisant desir " : CCCCIV 4 "faulx bourdon de maleur»; CCCCXX 5 "l'aumosne de liesse", 11 "repast que de foiblesse ", 12 "sur paille de destresse ": CCCCXXVIII 1-7-13 « ung sac de joyeuse promesse", 2-8 «en ung coffin d'oublie". Au total, donc, si l'on tient compte des reprises dans les refrains, quatre-vingt-seize occurrences. 
encore un transfert de propriétés. La particule DE, plutôt que constituer une marque syntaxique de processus, de transition, établit un rapport syntaxique unilatéral, sans aucune ambiguïté, qui permet de considérer ces séquences davantage comme des réifications que comme des métaphores de la dénotation ${ }^{48}$, et de les éditer comme telles avec une minuscule.

Il y a, ensuite, une deuxième catégorie de séquences qui relèvent à l'apparence d'un même champ d'images, mais qui sont soumises, en réalité, à deux processus métaphoriques bien distincts, que l'analyse des propriétés sémantiques des constituants peut seulement en partie aider à expliquer. D'une part, nous avons des séquences avec des substantifs concrets indiquant un lieu, un espace physique anthropomorphique ("jardin», "prison», "hermitage», etc.), souvent associés à des abstracta jouant d'habitude, en dehors de ces syntagmes, le rôle de personnification («Soussy», "Fortune», etc.). L'union de ces deux ou trois constituants (substantif concret + adjectif + substantif abstrait) crée un espace sémantique où l'homme semble pouvoir se mouvoir avec agilité entre abstrait et concret. En réalité, le contexte, le contexte aurélien tout entier, ne semble pas offrir d'actualisation poétique au processus de transfert des composantes sémantiques du sémème concret dans celles du terme abstrait et vice versa. En effet, il se trouve que, ou bien la symbolique de ces realia s'avère être obscure («cuevrechief de plaisance"), ou appauvrie par l'usage courtois ("yeulx de joyeuse plaisance "), ou bien que la subjectivisation des abstracta soit validée seulement partiellement par le contexte du rondeau ou de la ballade, ou encore qu'elle apparaisse comme invraisemblable ou irréelle

48. Armand Strubel pense " qu'il suffit d'un possessif pour démontrer la séquence allégorique et la restituer à la métaphore originelle : le second terme, privé de sa généralité, renvoie à une entité concrète ", c'est-à-dire actualisante (cf. A. Strubel, op. cit. p. 175). La remarque est sans doute intéressante, mais elle se prête à quelques observations. D'une part, elle n'est pas toujours applicable, comme semblerait le prouver le cas des différentes "forest de Longue Actente ", identiques sur le plan morphologique et syntaxique et pourtant si diverses sur le plan du contenu et de l'expression; d'autre part elle ne vise qu'une minorité de séquences (deux exactement : "mon livre de pensee " et "mon jardin de pensee»), qui semblent sollicitées par deux extrêmes, et qui pourraient aussi entrer dans le cas de la métaphore-personnification). 
(" nef de desireux vouloir ", « sentine d'alegeance ", etc.). La particule DE, alors, ne fait qu'instituer un rapport de soumission ou de possession, que l'on pourrait noter éditorialement en attribuant une minuscule au terme concret et une majuscule à celui abstrait, et en traitant, donc, ces séquences ${ }^{49}$ comme des cas particuliers de personnification.

D'autre part, ces mêmes substantifs concrets qui indiquent un espace physique ou conceptuel ( livre», «forest», " nef », etc.), combinés avec ces mêmes abstracta caractéristiques de la poétique aurélienne (Nonchaloir, Pensee, Esperance, etc.), ou avec des substantifs abstraits auxquels la tradition courtoise n'a jamais attribué la fonction d'abstractum agens, et dont le processus de subjectivisation s'explique aussi bien par des raisons endogènes au syntagme que contextuelles, subissent un phénomène, d'abord, de stérilisation de leurs propriétés connotatives, et ensuite de translation conceptuelle. Elles seraient, en d'autres mots, ce que les sémiologues appellent des cas de ratio difficilis, c'est-à-dire que ces syntagmes se posent comme des occurrences expressives qui concordent directement avec leur propre contenu, soit parce qu'il n'existe pas de type expressif préconçu, soit parce que celui-ci est une réplique du type de contenu. La particule DE dénote, ici, une marque de processus, de transfert catégoriel, ou de transfert des propriétés dénotatives, créant

49. Appartiendraient, donc, à ce type de personnifications les 46 occurrences suivantes : a) Ballades : VIII 4 «Ou rommant de Plaisant Penser »; XXVII 9-18-27-33 «En la prison de Desplaisance»; XXVIII 5-6 « au port / De Desir»; XXXIV 1 «l'uis de Pensee»; XLIII 2 « En l'ermitage de Pensee ", 9 " ou bois de Merencolie »; LXIII 1 " En la forest d'Ennuyeuse Tristesse"; XCVIII 19 «ou bateau du Monde"; CI 17-18 «en reclusaige/ De Desconfort et Douleur»; CII 12 "Au hault paradis de Plaisance"; CV 7 «En la cité de Destinee », 9 «L'ostellerie de Pensee»; b) Chansons: XVII 6 «d'Espoir le port»; XXIII 4 «du livre de Joie»; XLV 11 «le manoir de Joie"; Rondeaux : XXVI 6 " ou livre de Joie»; LXXXI 6 "la sentine d'Alegeance"; CXL 1-7-13 «l'abisme de Douleur»; CCXXXI 9 "l'uys de Pensee "; CCLVII 4 « Dedans mon jardin de Pensee"; CCLXXV 1-2-7-8-13-14 «en hermitage/De Nonchaloir»; CCLXXX 4 «la nef de Desireulx Vouloir »; CCLXXXV 2-8 « le molin de Pensee»; CCCXI 1-7-13 «le logeis de Joye ", 2-8 "L'ostellerie de Pensee"; CCCXXXII 4 «pays de Desirance", 12 "Au port de Bonne Destinee»; CCCLII 2 "Ou purgatoire de Soussy"; CCCLXXXIII 1-7-13 "des prisons de Pensee"; CCCCIV 12 «la chappelle de Douleur»; CCCCXXII 1-7-13 « la maison de Douleur». 
une dimension purement poétique, qui n'est accessible que par les sentiers du contexte, éclairés par le jeu des projecteurs rimiques et rythmiques (positionnement à la rime et à l'incipit-refrain), et par la proportion instituée entre les composantes sémantiques du sémème concret et celles du sémème abstrait. Le type expressif coïncide alors avec le sémème véhiculé par l'occurrence expressive dans sa totalité ${ }^{\text {so, }}$, qui nous apparaît alors comme une "singularité nue » ${ }^{51}$ avec une densité poétique infinie : un poème en un vers, en une image 52 .

Le caractère emblématique de ces métaphores créatives, que l'on pourrait ainsi définir comme métaphores de la dénotation, telles que "Forest de Longue Actente ", "Livre de Pensee », " Nef d'Esperance ${ }^{5.3}$, serait alors mis en relief par l'attribution de la majuscule à toute la séquence métaphorique, permettant, ainsi, de représenter aussi graphiquement, donc semiotiquement, la subjectivisation des abstracta et la symbolique des realia.

Quand on l'examine de près, un tel choix se révèle seulement à l'apparence fondée sur une répartition typologique a posteriori de ces séquences métaphoriques, puisqu'au Duc, lui-même, la plupart des assemblages du premier type ont dû apparaître comme forcés,

50. Sur les rapports entre type et occurrence, ratio facilis et ratio difficilis, on peut lire des pages éclairantes dans le livre d'Umberto Eco, La production des signes, Paris, 1992, en part. les p. 21-34.

51. J'emprunte cette définition aux physiciens Roger Penrose et Stephen Hawking qui l'ont utilisee pour décrire les singularités produites par les effondrements gravitationnels (les trous noirs). Il me semble, en effet, que ces séquences représentent la substance résiduelle d'un effondrement syntaxique et d'une condensation de la matière sémantique, où le poète a caché pudiquement, par une sorte de censure poétique, les impasses d'un monde et d'une vie.

52. Que l'on pense. par exemple, à l'Apollinaire de Chantre : «Et l'unique cordeau des trompettes marines », ou à l'Ungaretti de Mattina : "M'illumino / d'immenso ".

53. Devraient, donc, entrer dans le cas de la métaphore emblématique ou créative quatre séquences d'images, pour un total de douze occurrences : a) Ballades : XXVIII 1 «Nef de Bonne Nouvelle»; LXXV 15 « en la Nef d'Esperance»; CV 1 «en la Forest de Longue Actente»; b) Rondeaux : XXXIII 1-7-13 "Dedans mon Livre de Pensee"; CCXXV 1-9-15 «En la Forest de Longue Actente»; CCXXVIII 1-9-15 «En la Forest de Longue Actente ", auxquelles il conviendrait peut-être d'ajouter aussi «l'Hermitage de Nonchaloir ". 
artificiels, s'il ne les a utilisés qu'une seule fois, et surtout s'il les a laissés dériver dans ses poèmes sans autres amarres sémantiques et conceptuelles que celles du jeu allégorique. Quand on sait, au contraire, la place que joue dans la sélection des mots-clés, par conséquent dans leur production, le refrain, on ne peut pas ne pas remarquer que la plupart des séquences, que nous avons définies comme métaphores de la dénotation, apparaissent dans les poèmes à l'incipit-refrain (c'est le cas, notamment, du "Livre de Pensee ", de la "Nef de Bonne Nouvelle » et de la « Forest de Longue Actente »), ou au début de la strophe («Nef d'Esperance»), connotant ainsi tout le poème avec leur présence multipliée par les écho internes de la ritournelle.

Aristote pensait déjà que grâce aux métaphores nous connaissons quelque chose ${ }^{54}$ - où le verbe connaître veut indiquer aussi bien la production que la réception --, nous pouvons dire que c'est grâce à ces métaphores créatives, à ces métaphores de la dénotation, que Charles d'Orléans a découvert son monde intérieur en gravant ses états d'âme sur autant de clichés immaculés, parce que purifiés de toute incrustation connotative hors-textuelle. C'est pour cela que ces séquences d'images, au lieu de présupposer un contexte de référence et de relecture très vaste - comme devrait le faire, selon Umberto Eco, toute métaphore réussie --., et d'inférer un univers intertextuel donné - ce qui, d'ailleurs, est en partie vrai pour certaines d'entre elles 55 - incitent, par l'immobilité de leur champ d'images 56 (bildfeld), c'est-à-dire par leur non paraphrasabilité, à une réflexion ouverte non pas sur l'intertexte -- exercice d'interprétance aussi légitime qu'antiéconomique - mais sur le contexte, l'hic et nunc du poète, sa vision du monde : sa connaissance des choses.

Claudio Galderisi.

54. Cf. Aristote, Rhitorique, III, 10, 1410.

55. Armand Strubel a mis en évidence le caractère intertextuel d'énoncés comme "le livre de pensée ", et leur proximité avec des tournures du langage (ex. "le livre de raison", ou le "livre d'heures»), qui enlèverait à ces formules leur charge référentielle révolutionnaire (cf. A. Strubel, op. cit., p. 175).

56. Sur les champs d'images et le rapport entre métaphore et sens, voir les travaux de Weinrich (cf. H. Weinrich, Münze und Wort. Untersuchungen an einem Bildfeld, dans Romanica. Festschrift Gerhard Rohlfs, 1957, p. 508-521). 